\title{
Image-based modeling of inhomogeneous single-scattering participating media
}

\author{
HU Yong
}

State key Laboratory of Virtual Reality Technology and Systems, Beihang Universty, Beijing 100191, China

The State Key Laboratory of Virtual Reality Technology and Systems, Beihang University in Beijing, has proposed an image based modeling method for inhomogeneous single-scattering participating media whose density fields may contain high frequency details. This work could reduce the difficulty in capturing real-world participating media, which can be applied to 3D games, film production, virtual reality systems, etc. This study is reported in Volume 53 (June, 2010) of the Science China: Information Sciences because of its significant research value.

Realistic modeling of real-world objects is one of the most important topics in the area of Virtual Reality. Currently, geometric modeling of solid objects has made much headway, whilst the modeling of dynamic and fluidic participating media is less studied. Rich visual effects can be produced through the interaction between participating media and lighting, such as a scene shrouded with mist, an arena with smoke and various lighting sources coexisting, or the process of red wine or milk diffusing in water, etc. Therefore, a model for realistic participating media is of great value in enhancing immersion into a virtual environment. The propagation of light in participating media is a very complex process, involving three phenomena: scattering, absorption and media self-emission. The intensity perceived by one's line of sight is the integration of the intensities at all positions along the line within the media that traverses all three phenomena. As it is very difficult to construct the dynamic density field of inhomogeneous participating media and make realistic visual effects with traditional methods (e.g., using particle systems and hydrodynamic equations), the modeling of participating media is an extremely difficult issue in Virtual Reality and Computer Graphics. Currently, the rapid development of digital imaging technology makes it possible to fabricate devices for capturing the real interaction process between lighting and participating media. The fabrication of devices and the use of image-based modeling methods are the main directions of the related research.

So far, related work that can reconstruct the participating media with high frequency detail needs to make good use of complex optical devices such as lasers and high-speed projectors. We focus on proposing an image based modeling method in which the inputs are only a few images. Therefore, in this work, we propose a method to reconstruct inhomogeneous single-scattering participating media, which could preserve the fine high-frequency details of the density fields. Volumetric data and the ratio of the absorption coefficient to the scattering coefficient are used to describe the spatial-varying density distribution and optical properties of certain participating media, and a function relating the above parameters and the captured pixel values is developed. Thus, the problem of how to find solutions to these parameters is formulated as a nonlinear numerical optimization problem. To reduce large time overheads and numerical instability brought about by simultaneously solving large numbers of voxels, we propose an initialization algorithm for enabling the assigned density values to satisfy the regularity of brightness distribution in captured images, in a manner that is nearly as good as the progressive refinement algorithm for multi-resolution volumetric data. Moreover, we propose a parallel multi-voxel gradient computation algorithm using hardware acceleration to reduce the time overheads in the gradient computation of large numbers of voxels.

The authors are affiliated to the State Key Laboratory of Virtual Reality Technology and Systems, Beihang University. This laboratory is conducting research mainly in four areas: Modeling Theory and Methodology in Virtual Reality, Augmented Reality and Human-computer Interaction Mechanisms, Distributed Virtual Reality Methods and Techniques, and Virtual Reality Platform Tools and Systems. The third author of this paper is affiliated to Microsoft Research Asia.

Funding from the National Natural Science Foundation of China (Grant No. 60773153), National Hi-Tech Research Program of China (Grant No. 2009AA012102) and the Beijing Natural Science Foundation (Grant No. 4102037) supported this research.

See the articles: Hu Y, Qi Y, Tong X. Image-based modeling of inhomogeneous single-scattering participating media. Sci China Inf Sci, 2010, 53:1141-1150, doi:10.1007/s11432-010-0104-y

Zhao Q P. A survey on virtual reality. Sci China Ser F-Inf Sci, 2009, 52: 348-400

Qi Y, Yang S, Cai S, et al. A method of 3D modeling and codec. Sci China Ser F-Inf Sci, 2009, 52: 758-769

Siegel R, Howell J R. Thermal Radiation Heat Transfer. 2nd ed. Washington: Hemisphere Pub. Corp., 1981. 412-484 